\title{
Limits of serum carcinoembryonic antigen (CEA) and carbohydrate antigen (CA) 19-9 as the diagnosis of gallbladder cancer
}

\author{
Jae Seung KANG, Yoo Jin CHOI, Yoonhyeong BYUN, Youngmin HAN, Jung Min LEE, \\ Hee Ju SOHN, Hongbeom KIM, Wooil KWON, Jin-Young JANG* \\ Surgery and Cancer Research Institute, Seoul National University College of Medicine, Seoul, Korea
}

Introduction: Because the clinical symptoms of gallbladder (GB) cancers are lacking or vague, objective tools for diagnosing GB cancer were necessary. Although serum carcinoembryonic antigen (CEA) and carbohydrate antigen (CA) 19-9 were widely utilized for the diagnosis of GB cancer, few studies existed about the diagnostic performance of these biomarkers. This study aimed to investigate the diagnostic performance of serum CEA and CA 19-9, and demonstrate the clinical usefulness of them in diagnosing GB cancer.

Methods: This was a retrospective cohort study. Between January 2000 and March 2020, total 751 GB cancer patients and 2,310 normal controls were included. Two biomarkers were measured before surgery. Receiver operating characteristics curves were obtained, and sensitivity and specificity of each biomarker were evaluated. Cancer stage was unified to American Joint Committee on Cancer 7 th staging system.

Results: 666 (88.8\%) patients had serum CEA $\leq 5 \mathrm{ng} / \mathrm{mL}$, and $546(72.7 \%)$ patients had serum CA 19-9 $\leq 37 \mathrm{IU} / \mathrm{mL}$. In terms of differentiating the GB cancer from the control, the sensitivity and specificity of serum CEA at $5 \mathrm{ng} / \mathrm{mL}$ was $11.2 \%$ and $99.2 \%$, and those of serum CA $19-9$ at $37 \mathrm{IU} / \mathrm{mL}$ was $27.3 \%$ and $94.6 \%$. When these cut-off values were applied to differentiate early GB cancer from advanced tumor, the sensitivity and specificity at these cut-off values were $14.2 \%$ and $96.1 \%$ in CEA and $33.6 \%$ and $90.1 \%$ in CA $19-9$.

Conclusions: Serum CEA and CA 19-9 were not suitable for screening GB cancer patients from controls. Two biomarkers should be replaced to new biomarkers with higher. 